\title{
Chair side measuring instrument for quantification of the extent of a transverse maxillary occlusal plane cant
}

\author{
Farhad B. Naini ${ }^{1,2^{*}}$ D, Ashraf Messiha ${ }^{2}$ and Daljit S. Gill ${ }^{3}$
}

\begin{abstract}
Background: Treatment planning the correction of a transverse maxillary occlusal plane cant often involves a degree of qualitative "eyeballing", with the attendant possibility of error in the estimated judgement. A simple chair side technique permits quantification of the extent of asymmetry and thereby quantitative measurements for the correction of the occlusal plane cant.

Methods: A measuring instrument may be constructed by soldering the edge of a stainless steel dental ruler at $90^{\circ}$ to the flat surface of a similar ruler. With the patient either standing in natural head position, or alternatively seated upright in the dental chair, and a dental photographic retractor in situ, the flat under-surface of the horizontal part of this measuring instrument is placed on a unilateral segment of a bilateral structure, e.g. the higher maxillary canine orthodontic bracket hook. The vertical ruler is held next to the contralateral canine tooth, and the vertical distance measured directly from the canine bracket to the flat under-surface of the horizontal part of the measuring instrument.
\end{abstract}

Results: This vertical distance quantifies the overall extent of movement required to level the maxillary occlusal plane. Conclusions: This measuring instrument and simple chair side technique helps to quantify the overall extent of surgical levelling required and may be a useful additional technique in our clinical diagnostic armamentarium.

Keywords: Transverse cant, Occlusal plane, Orthognathic surgery, Symmetry

\section{Background}

Relative unilateral vertical over- or underdevelopment of the maxilla and maxillary dentoalveolus leads to a transverse cant of the maxillary occlusal plane [1]. Correction of such a cant requires a Le Fort I level osteotomy, followed by unilateral bone removal and superior repositioning, contralateral inferior repositioning and bone grafting, or a combination of the two, in order to level the maxillary occlusal plane [2]. The degree of unilateral superior versus contralateral inferior repositioning depends on the aesthetic parameters of maxillary incisor exposure in repose and overall maxillary dentogingival exposure on smiling $[1,2]$. Accurate planning to correct

\footnotetext{
* Correspondence: farhad.naini@yahoo.co.uk

'Kingston and St George's Hospitals and St George's Medical School, London, UK

${ }^{2}$ Maxillofacial Unit, St George's Hospital Medical School, Blackshaw Road, London SW17 OQT, UK

Full list of author information is available at the end of the article
}

such an asymmetry is paramount [3-6]. The following instrument and chair side technique to quantify the extent of asymmetry have not been previously described.

\section{Methods}

A measuring instrument may be constructed by soldering the edge of a double-sided stainless steel dental ruler at $90^{\circ}$ to the flat surface of a similar ruler (Fig. 1). With the patient in natural head position, near a vertical plumb line (forming a true vertical line), and a dental photographic retractor in situ (Fig. 2), the flat under-surface of the horizontal part of this measuring instrument is placed on the higher maxillary canine orthodontic bracket hook and held perpendicular to the true vertical (Fig. 3). The vertical ruler is held next to the contralateral canine tooth, and the vertical distance measured directly from the canine bracket to the flat under-surface of the horizontal part of the measuring instrument. Alternatively, if the patient is 


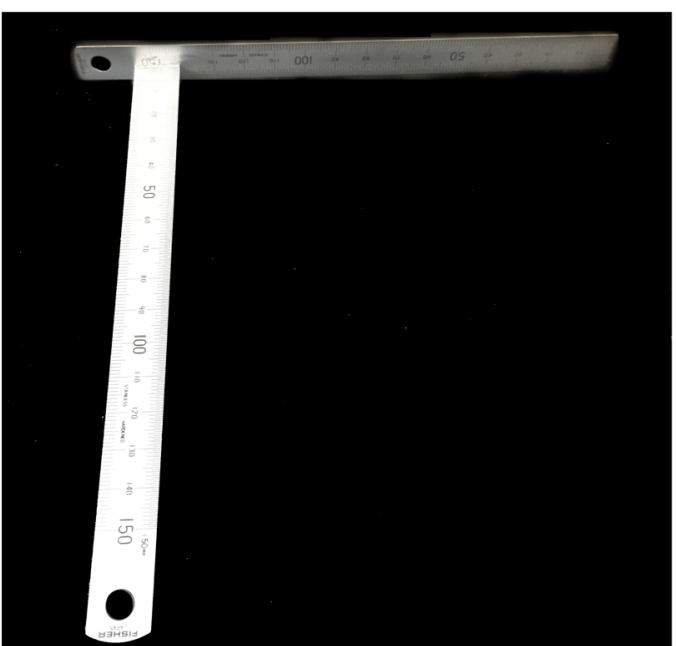

Fig. 1 The measuring instrument is constructed by soldering the edge of a double-sided stainless steel dental ruler at $90^{\circ}$ to the flat surface of a similar ruler. A double-sided ruler permits its use on the patient's right and left sides as required. A right-angle gauge may be used to ensure a $90^{\circ}$ angle

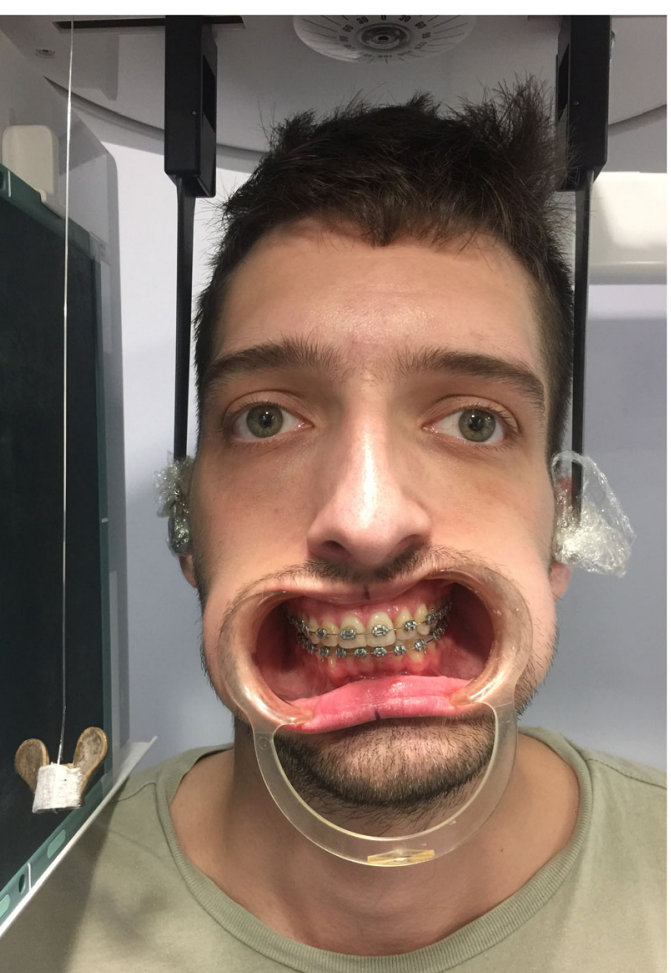

Fig. 2 Patient is shown in natural head position. The oral retractors are in situ, and a plumb line is evident hanging to the patient's right side, which acts as a guide to the true vertical line. A transverse cant of the maxillary occlusal plane, down on the patient's right side, is evident. The patient may be positioned in a cephalostat as demonstrated here, though this is not mandatory, and for most patients sitting in the dental chair will suffice

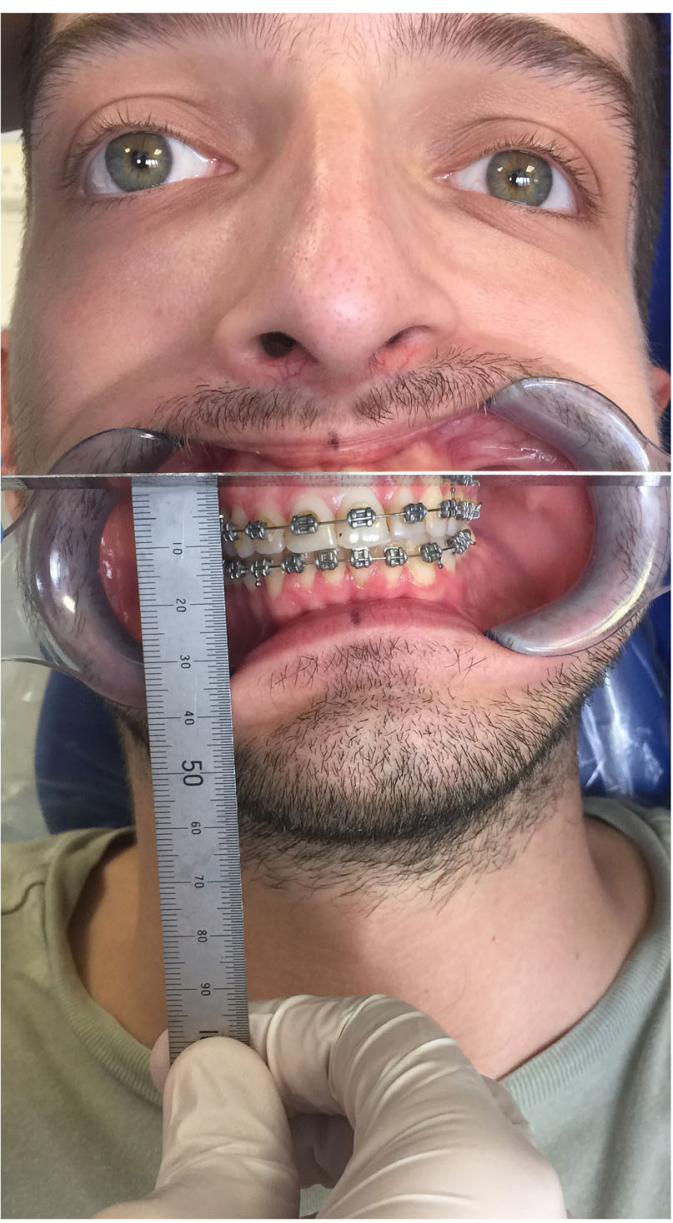

Fig. 3 The flat under-surface of the horizontal part of the measuring instrument is placed on the higher maxillary canine orthodontic bracket hook (though it may be placed on a unilateral segment of any bilateral structure), and held perpendicular to the true vertical. For patients with a relatively symmetrical upper face, and no vertical orbital dystopia, the horizontal part of the measuring instrument may be held approximately parallel to the interpupillary plane (a dental mirror handle may be held in line with the interpupillary plane, to aid visualisation at the chair side). The vertical ruler is held next to the contralateral canine tooth, and the vertical distance measured directly from the canine bracket to the flat undersurface of the horizontal part of the measuring instrument. This vertical distance quantifies the overall extent of movement required to level the maxillary occlusal plane

seated upright in the dental chair, the horizontal part of the instrument may be held parallel to the interpupillary line, assuming no vertical orbital dystopia is evident.

It is worth emphasising that the instrument may be held relative to a true vertical plumb line hanging from the ceiling if a patient has a severe craniofacial asymmetry and vertical orbital dystopia. However, this is usually not the case in orthognathic patients, and we suggest that when the interpupillary line is essentially parallel (i.e. the absence of vertical orbital dystopia), then the horizontal part of the instrument may be help parallel to the interpupillary line. 


\section{Results}

The vertical distance thus measured quantifies the overall extent of movement required to level a transversely canted maxillary occlusal plane.

\section{Discussion}

One of the key principles in planning the correction of significant dentofacial asymmetries is levelling of the maxillary occlusal plane [1]. This decision is made primarily based on the aesthetic parameter of the maxillary incisor and canine exposure in relation to the upper lip in repose, and the degree and symmetry of the exposure of the maxillary dentition and gingivae in animation $[1,2]$. If incisor and canine exposure is reduced, unilateral setdown of the maxilla may be required, albeit bearing in mind lower face height proportion and implications for surgical stability. Conversely, if dentogingival exposure is increased unilaterally, then ipsilateral maxillary impaction is the treatment of choice. The degree of impaction versus setdown required to accurately level the maxillary occlusal plane while maintaining or improving dentogingival aesthetics requires accurate planning. In addition to the important clinical and cephalometric techniques required for precision, the simple chair side technique potentially improves visualisation and permits an additional verification to aid both diagnosis and treatment planning for such patients.

\section{Conclusions}

This measuring instrument and simple chair side technique helps to quantify the overall extent of surgical levelling required and may be a useful additional technique in our clinical diagnostic armamentarium.

\section{Acknowledgements}

Nil.

Funding

None.

Availability of data and materials

Not applicable.

\section{Authors' contributions}

FBN conceived the idea, which was deliberated with AM and DSG and subsequently clinically verified by all authors. All authors helped to complete the manuscript and read and approved the final manuscript.

Ethics approval and consent to participate

Ethical approval was not required.

Consent for publication

The subject in Figs. 2 and 3 provided written consent for his images to be published.

Competing interests

The authors declare that they have no competing interests.

\section{Publisher's Note}

Springer Nature remains neutral with regard to jurisdictional claims in published maps and institutional affiliations.

\section{Author details}

'Kingston and St George's Hospitals and St George's Medical School, London, UK. 'Maxillofacial Unit, St George's Hospital Medical School, Blackshaw Road, London SW17 0QT, UK. ³ Department of Orthodontics, Great Ormond Street Hospital \& Eastman Dental Hospital, London, UK.

Received: 27 March 2019 Accepted: 15 April 2019

Published online: 15 May 2019

\section{References}

1. Naini FB (2011) Facial symmetry and asymmetry. In: Naini FB. Facial aesthetics: concepts and clinical diagnosis. Wiley-Blackwell, Oxford

2. Naini FB, Manisali M, Gill DS (2017) Asymmetries of the maxilla and mandible. In: Naini FB, Gill DS (eds) Orthognathic surgery: principles, planning and practice. Wiley-Blackwell, Oxford

3. Quevedo L, Ruiz JV, Quevedo C (2011) Using a clinical protocol for orthognathic surgery and assessing a 3-dimensional virtual approach: current therapy. J Oral Maxillofac Surg 69:623-637

4. Xia JJ, Gateno J, Teichgraeber JF (2009) New clinical protocol to evaluate craniomaxillofacial deformity and plan surgical correction. J Oral Maxillofac Surg 67:2093-2106

5. Barbenel JC, Paul PE, Khambay BS, Walker FS, Moos KF, Ayoub AF (2010) Errors in orthognathic surgery planning: the effect of inaccurate study model orientation. Int J Oral Maxillofac Surg 39:1103-1108

6. Zhao L, Patel PK, Cohen M (2012) Application of virtual surgical planning with computer assisted design and manufacturing technology to craniomaxillo-facial surgery. Arch Plastic Surg 39:309-316

\section{Submit your manuscript to a SpringerOpen ${ }^{\circ}$ journal and benefit from:}

- Convenient online submission

- Rigorous peer review

- Open access: articles freely available online

High visibility within the field

- Retaining the copyright to your article

Submit your next manuscript at $\boldsymbol{\nabla}$ springeropen.com 